\title{
Fuzzy physical programming for Space Manoeuvre Vehicles trajectory optimization based on hp-adaptive pseudospectral method
}

\author{
Runqi Chai ${ }^{\mathrm{a}}$, Al Savvaris ${ }^{\mathrm{a}}$, Antonios Tsourdos $^{\mathrm{a}}$ \\ ${ }^{a}$ School of Aerospace, Transport and Manufacturing, Cranfield University, Bedfordshire, \\ MK43 OAL, United Kingdom
}

\begin{abstract}
In this paper, a fuzzy physical programming (FPP) method has been introduced for solving multi-objective Space Manoeuvre Vehicles (SMV) skip trajectory optimization problem based on hp-adaptive pseudospectral methods. The dynamic model of SMV is elaborated and then, by employing hp-adaptive pseudospectral methods, the problem has been transformed to nonlinear programming (NLP) problem. According to the mission requirements, the solutions were calculated for each single-objective scenario. To get a compromised solution for each target, the fuzzy physical programming (FPP) model is proposed. The preference function is established with considering the fuzzy factor of the system such that a proper compromised trajectory can be acquired. In addition, the NSGA-II is tested to obtain the Pareto-optimal solution set and verify the Pareto optimality of the FPP solution. Simulation results indicate that the proposed method is effective and feasible in terms of dealing with the multi-objective skip trajectory optimization for the SMV.
\end{abstract}

Keywords: Space Manoeuvre Vehicles, fuzzy physical programming, hp-adaptive pseudospectral, nonlinear programming, multi-objective.

\section{Introduction}

Over the past couple of decades, trajer ory optimization problems in terms of reentry vehicle [1, 2, 3, 4, 5, 6, have attracted significant attention. One of the

Email addresses: r.chai@cranfield.ac.uk (Runqi Chai), a.savvaris@cranfield.ac.uk (Al Savvaris)

2017年3月23日

Published by Elsevier. This is the Author Accepted Manuscript issued with: Creative Commons Attribution Non-Commercial No Derivatives License (CC:BY:NC:ND 3.0). The final published version (version of record) is available online at DOI:10.1016/j.actaastro.2016.02.020. Please refer to any applicable publisher terms of use. 
current objectives is the development of Space Manoeuvre Vehicles(SMV) for a

5 dynamic mission profile. The Mach number and the flight altitude of the reentry vehicle vary largely during the whole reentry phase, the aerodynamic feature of the vehicle has large uncertainties and nonlinearities. Due to these reasons, the use of numerical methods to handle these types of problems is commonly used. Numerical methods for solving optimal control problems are divided into two major classes: indirect methods and direct methods 7, 8, 9, 10. However, it is very difficult to solve the trajectory design problem by using indirect methods based on maximum principle. Therefore, direct optimization method has been widely used for trajectory optimization. Applying direct methods meant the development of several discrete methods[11].

In recent years, collocation methods for transforming optimal control problems have increased in popularity [12, 13. There are two main kinds of collocation methods, local collocation method such as the direct collocation and global collocation method e.g. the pseudospectral [14, 15, 16]. In a pseudospectral method, the collocation points are based on quadrature rules and the basis function are Lagrange or Chebyshev polynomials. In contrast to the direct collocation method, pseudospectral method usually divides the whole time history into a single mesh interval whereas its counterpart, direct collocation, divides time interval into several equal step subintervals and the convergence is achieved by adding the degree of the polynomial. To improve accuracy and computation-

25 al efficiency using pseudospectral method, L. Darby presented a hp-strategy in [17, 18, 19. By adding collocation points in a certain mesh interval or dividing the current mesh into subintervals simultaneously, the accuracy of interpolation can be improved dramatically.

Generally, the traditional trajectory design usually aims at one single objective, for example, minimizing the aerodynamic heating, maximizing the cross range, etc. However, in reality, for space vehicle trajectory design, most the missions contain more than one requirements and this brings the developmen$\mathrm{t}$ of multi-objective optimization(MOO) [20]. There are many multi-objective methods which are suitable for these kind of problems. Commonly, the method only one single objective but it is difficult to determine the weight coefficients. In 1996, Messac designed a physical programming(PP) method to convert the objectives [21, 22], which removes the information of priority and weight coeffi- 
cients. But in practice, usually there are some fuzzy factors in the real system and because of this, a fuzzy physical programming method is proposed in this paper.

The mission scenario investigated in this paper focuses on the atmospheric skip hopping, targeting the entry into the atmosphere down to a predetermined position (predetermined altitude given by the industrial sponsor of this project) and the required controls involved in returning back to low earth orbit. Studies can be found in the literature regarding the skip reentry of deep-space spacecraft with high speed over first cosmic velocity, however in the scenario considering in this paper, a high thrust engine would be necessary for SMV to return to low earth orbit. The overall mission can be found in Fig.1. General skip reentry can

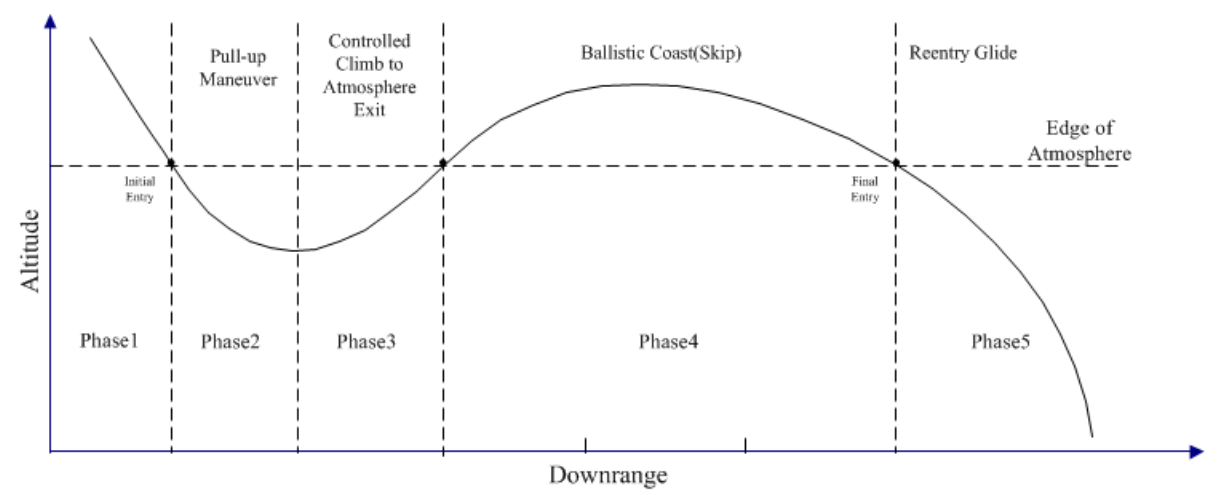

Figure 1: Mission profile

be divided into five phases: initial roll, down control, up control, Kepler and final entry. Considering the mission of the SMV is to overfly the ground target with specific altitude, the most challenging phase 2 and 3 will be considered in this paper.

Most of the current studies in trajectory optimization are based on the numerical simulation. Smirnov et al. 23] 24, presented studies in terms of developing mathematical model for evaluation of stochastic numerical errors accumulation. Based on the published simulation results, the problem of accumulation of errors cannot be ignored. Therefore, the effect of noise on the trajectory optimization is also considered in this work, and the results are presented in 60 Section 5 of this paper.

The paper is organised as follows. In section 2 , we introduce the aerodynam- 
ic model of the SMV reentry vehicle and some basic principles of the trajectory optimization problem. Section 3 describes the method used to discretize the optimal control problem. Then in section 4 the fuzzy physical programming(FPP)

${ }_{65}$ procedures of solving multi-objective SMV trajectory problem is detailed. Following that, section 5 present comparison between solution calculated for each single-objective and the compromised solution generated by employing the FPP approach.

\section{Problem Description}

\subsection{SMV dynamic model}

The Earth is considered as a symmetrical sphere and the earth rotation is ignored. Considering a three degree of freedom dynamic equations of SMV reentry vehicle:

$$
\begin{aligned}
\dot{r} & =V \sin \gamma \\
\dot{\theta} & =\frac{V \cos \gamma \sin \psi}{r \cos \phi} \\
\dot{\phi} & =\frac{V \cos \gamma \cos \psi}{r} \\
\dot{V} & =\frac{T \cos \alpha-D}{m}-g \sin \gamma \\
\dot{\gamma} & =\frac{L \cos \sigma+T \sin \alpha}{m V}+\left(\frac{V^{2}-g r}{r V}\right) \cos \gamma \\
\dot{\psi} & =\frac{L \sin \sigma}{m V \cos \gamma}+\frac{V}{r} \cos \gamma \sin \psi \tan \phi \\
\dot{m} & =-\frac{T}{I_{s p} g} \\
\dot{\alpha} & =K_{\alpha}\left(\alpha_{c}-\alpha\right) \\
\dot{\sigma} & =K_{\sigma}\left(\sigma_{c}-\sigma\right) \\
\dot{T} & =K_{T}\left(T_{c}-T\right)
\end{aligned}
$$

where $r$ is the radial distance from the Earth center to the vehicle, $\theta$ and $\phi$ are the longitude and latitude, $V$ is the Earth-relative velocity, $\gamma$ is the relative flight-path angle, $\psi$ is the relative velocity heading angle measured clockwise from the north, $m$ is the mass of the vehicle, $t$ is time, control variables are angle of attack $\alpha_{C}$, bank angle $\sigma_{C}$ and thrust $T_{C}$, respectively. In reality, the real control variables cannot change dramatically (i.e. from lower bound to 
upper bound). Therefore, in the model provided (1), three rate constraints are introduced by using the technique of first order lag which can be concluded to the last three equations in (1).

The atmosphere model, lift $L$ and $\operatorname{drag} D$ can be defined as:

$$
\begin{array}{ll}
g=\frac{\mu}{r^{2}} & \rho=\rho_{0} \exp \frac{r-r_{0}}{h_{s}} \\
L=\frac{1}{2} \rho V^{2} C_{L} S & D=\frac{1}{2} \rho V^{2} C_{D} S
\end{array}
$$

where $S=2690 \mathrm{ft}^{2}$ is reference area, $\mu=1.4076539 \times 10^{16} \mathrm{ft}^{3} / \mathrm{s}^{2}$ is gravitational parameter of the earth. $\rho$ is density of atmosphere and $\rho_{0}=0.002378 \mathrm{slug} / \mathrm{ft}^{3}$ is density of atmosphere at sea-level. $r_{0}=20902900 \mathrm{ft}$ is Earth' s radius, $C_{L}$ and $C_{D}$ are lift and drag coefficient determined by angle of attack $\alpha$ and $M a$, respectively, $g$ is gravity acceleration.

The drag and lift coefficient can be determined by the following equations:

$$
\begin{aligned}
& C_{D}=C_{D 0}+C_{D 1} \alpha+C_{D 2} \alpha^{2} \\
& C_{L}=C_{L 0}+C_{L 1} \alpha
\end{aligned}
$$

where $C_{L 0}=-0.2070, C_{L 1}=1.676, C_{D 0}=0.07854, C_{D 1}=-0.3529, C_{D 2}=$ 2.040 .

\subsection{Reentry process constraints}

SMV reentry process should satisfy some constraints due to safety reasons and also depending on the mission requirements. These constraints can be summarised as initial and terminal constraints, path constraints and boundary constraints.

\subsubsection{Initial and terminal constraints}

The complete mission can be divided into two phases, the descent phase and exit phase. Due to the mission requirement, the state variables at minimum decent point are specified. The initial conditions of all the states are:

$$
[r, \phi, \theta, V, \gamma, \psi, m, \alpha, \sigma, T]=\left[r_{0}, \phi_{0}, \theta_{0}, V_{0}, \gamma_{0}, \psi_{0}, m_{0}, \alpha_{0}, \sigma_{0}, T_{0}\right]
$$

On the other hand, at the minimum altitude point and final point(i.e. final point to return back into low earth orbit), hence complete one hop, the terminal altitude constraints are:

$$
[r(1), r(f)]=\left[r_{b}, r_{f}\right]
$$




\section{respectively.}

\subsubsection{Path constraints}

During the whole time period, to protect the structure of reentry vehicle, in simulation the SMV model need to satisfy strict path constraint, which can be summarised as follows:

$$
\begin{gathered}
\dot{Q}_{d}=K_{Q} \rho^{0.5} V^{3.07}\left(c_{0}+c_{1} \alpha+c_{2} \alpha^{2}+c_{3} \alpha^{3}\right)<Q_{d \max } \\
P_{d}=\frac{1}{2} \rho V^{2}<P_{d \max } \\
n_{L}=\frac{\sqrt{L^{2}+D^{2}}}{m g}<n_{\text {Lmax }}
\end{gathered}
$$

where $c_{0}=1.067, c_{1}=-1.101, c_{2}=0.6988, c_{3}=-0.1903$ and $K_{Q}=9.289 \times$ $10^{-9} \mathrm{Btu} \cdot \mathrm{s}^{2.07} / \mathrm{ft}^{3.57} / \mathrm{slug} \mathrm{g}^{0.5}$. $Q_{\text {dmax }}, P_{\text {dmax }}$ and $n_{\text {Lmax }}$ represents allowable maximum heating rate, dynamic pressure and acceleration, respectively.

\subsubsection{Boundary constraints}

For the SMV, the states should be limited as:

$$
\begin{array}{ll}
r_{\min } \leq r \leq r_{\max } & \theta_{\min } \leq \theta \leq \theta_{\max } \\
\phi_{\min } \leq \phi \leq \phi_{\max } & V_{\min } \leq V \leq V_{\max } \\
\gamma_{\min } \leq \gamma \leq \gamma_{\max } & \psi_{\min } \leq \psi \leq \psi_{\max } \\
m_{\min } \leq m \leq m_{\max } & \alpha_{\min } \leq \alpha \leq \alpha_{\max } \\
\sigma_{\min } \leq \sigma \leq \sigma_{\max } & T_{\min } \leq T \leq T_{\max }
\end{array}
$$

and the boundaries in terms of the control variables are defined as:

$$
\begin{aligned}
\alpha_{c(\min )} & \leq \alpha_{c} \leq \alpha_{c(\max )} \\
\sigma_{c(\min )} & \leq \sigma_{c} \leq \sigma_{c(\max )} \\
T_{c(\min )} & \leq T_{c} \leq T_{c(\max )}
\end{aligned}
$$

\subsection{Objective function}

To ensure the SMV has enough fuel carry-out several skip loops and maximise the number of hops, the first objective would be to minimize the fuel consumption, i.e., maximize final mass value, during the whole process. Moveover, the total aerodynamic heating is very important and it can have a serious implications on the SMV integrity structure. In addition, it is not desirable to have too many oscillations during the mission as it will also impact the integrity of the structure. On the other hand, a high final velocity will provide more 
kinetic energy for the vehicle and hence reduce the overall mission time which is desirable. Therefore, the objective functions are selected in the analysis.

1). Maximizing the final mass:

$$
\max J_{1}=m\left(t_{f}\right)
$$

2). Minimizing the total aerodynamic heating:

$$
\min J_{2}=\int_{t_{0}}^{t_{f}} \dot{Q(t)} d t
$$

3). Minimizing the oscillation:

$$
\min J_{3}=\int_{t_{0}}^{t_{f}} \gamma(t)^{2} d t
$$

4). Maximizing the final velocity:

$$
\max J_{4}=V\left(t_{f}\right)
$$

5). Minimizing the final time:

$$
\min J_{5}=t_{f}
$$

By setting the cost function described in Eq. (9)-Eq. (13), the SMV trajectory problem can be considered as an optimal control problem which has minimum or maximum cost function value and satisfy the initial and terminal state constraints, control variable constraints, three path constraints and dynamic equations.

\section{Global collocation method}

To solve the problem using the numerical method, the trajectory design problem needs to be transformed to nonlinear programming(NLP) and the basic method used in this paper is the pseudospectral method. Compared to local direct collocation, it uses the initial time point $t_{0}$ and terminal time point $t_{f}$ as two node points and removes numbers of small time segment introduced in direct collocation method. In other words, by using pseudospectral method, there is only one time interval $\left[t_{0}, t_{f}\right]$ and can use orthogonal polynomial to 
Assuming the time interval of an optimal control problem is $\left[t_{0}, t_{f}\right]$, the pseudospectral method must be used on the $[-1,1]$ and therefore, we transform the time interval by using:

$$
t=\frac{t_{f}-t_{0}}{2} \tau+\frac{t_{f}+t_{0}}{2}
$$

Following the transformation of the time interval to the values $[-1,1]$, the next step is to generate the approximation of state and control. In the Pseudospectral method, the state and control of an optimal control problem are approximated as:

$$
\begin{aligned}
& x(\tau) \approx X(\tau)=\sum_{0}^{N} X_{i} L_{i}(\tau) \\
& u(\tau) \approx U(\tau)=\sum_{0}^{N} U_{i} L_{i}(\tau)
\end{aligned}
$$

where $\tau \in[-1,1], L_{i}(\tau),(i=0, \ldots, N)$ are the collocation points and a basis of Lagrange polynomials, respectively. The LGR points are used as the collocation point. LGR points are the root of linear combination of Legendre polynomials which can be written as:

$$
P_{K-1}(\tau)+P_{K}(\tau)=0
$$

where the $K$ th order Legendre polynominal $P_{K}(\tau)$ is

$$
P_{K}(\tau)=\frac{1}{2^{K} K !} \frac{d^{K}}{d \tau^{K}}\left[\left(\tau^{2}-1\right)^{K}\right]
$$

In order to improve the performance of global pseudospectral method, hpstrategy has been developed for mesh refinement. The goal of the hp-adaptive algorithm is to improve the accuracy of the solution in an effective manner by judging if a certain mesh interval has met a specified tolerance. If the mesh cannot meet the accuracy tolerance, then the number of nodes or intervals should be changed either by increasing the degree of the polynomial in the mesh interval or dividing the mesh into several segments.

Let $e_{\max }$ mean the maximum error at $i t h$ collocation point. If $e_{\max }$ can satisfy the tolerance, then can stop the iteration since collocation points in the $k t h$ interval can reach the tolerance. Otherwise, it should be divided into subintervals or more collocation points are added. 
Whether we need to divide the interval into segments or add collocation overpass the tolerance, then the trajectory in the current interval is very noisy and it should be divided into new subintervals. On the other hand, if the tolerance can be satisfied, meaning the trajectory is flat in current interval and the accuracy can be improved by adding more collocation points.

By using the approach described above, the dynamic model can be transformed to algebraic constraints. Combining with the constraints mentioned in section 2.2 and cost function presented in section 2.3, the SMV trajectory problem is converted to a nonlinear programming problem(NLP) with state and control variables at collocation points.

\section{Fuzzy physical programming}

The multi-objective optimization problem of SMV trajectory design can be solved using the Fuzzy Physical Programming(FPP) method. FPP involves converting a multi-objective problem into a single objective problem using fuzzy performance functions that capture the decision maker's preferences, and after that solving this transformed single-objective optimization to find a compromise solution.

\subsection{Physical programming}

To generate a preferred compromise during multi-objective system optimization, a method called physical programming $(\mathrm{PP})$ is introduced. The way that PP captures the designer's preferences is by using preference functions. Compared with other multi-objective methods, the application of PP does not require the decision maker to specify weights for different object functions. Rather, the decision maker needs to define ranges of differing degrees of desirability for each objective function. It has been shown that PP offers the user several advantages, for example, it can reduce computational effort and time, and at the same time, eliminate iterative selection of weights and priorities of objective functions.

The objective functions are classified into four types:

(i) Class 1-S: smaller-is-better(minimization).

(ii) Class 2-S: larger-is-better(maximization). 
(iii) Class 3-S: value-is-better(seek value).

(iv) Class 4-S: range-is-better(seek range).

Take class 1-S as an example, the boundary of the preference region is represented by some values of objective function and there are six ranges for for class 2-S is the mirror image of class 1-S.

$$
p_{i}^{k}=A_{0}\left(\xi_{i}^{k}\right) p_{i(k-1)}+A_{1}\left(\xi_{i}^{k}\right) p_{i k}+\overline{A_{0}}\left(\xi_{i}^{k}, \lambda_{i}^{k}\right) s_{i(k-1)}+\overline{A_{1}}\left(\xi_{i}^{k}, \lambda_{i}^{k}\right) s_{i k}
$$


where $\xi_{i}^{k}=\left(f_{i}-f_{i(k-1)}\right) /\left(f_{i k}-f_{i(k-1)}\right)$ and $0<\xi_{i}^{k}<1, \lambda_{i}^{k}=\left(f_{i k}-f_{i(k-1)}\right), k$ is the number of region and $k=2,3,4,5$. As can be seen from Eq. 19p, for each region, the preference function takes the form of a spline segment which can be defined by its value and slope.

$$
\begin{gathered}
s_{i k}=\left.\frac{\partial p_{i}^{k}}{\partial f_{i}^{k}}\right|_{f_{i}^{k}=f_{i k}} \\
A_{0}(\xi)=\frac{1}{2} \xi^{4}-\frac{1}{2}(\xi-1)^{4}-2 \xi+\frac{3}{2} \\
A_{1}(\xi)=-\frac{1}{2} \xi^{4}-\frac{1}{2}(\xi-1)^{4}+2 \xi-\frac{1}{2} \\
\overline{A_{0}}(\xi, \lambda)=\lambda\left[\frac{1}{8} \xi^{4}-\frac{3}{8}(\xi-1)^{4}-\frac{1}{2} \xi+\frac{3}{8}\right] \\
\overline{A_{1}}(\xi, \lambda)=\lambda\left[\frac{3}{8} \xi^{4}-\frac{1}{8}(\xi-1)^{4}-\frac{1}{2} \xi+\frac{1}{8}\right]
\end{gathered}
$$$$
230
$$

In the end, for the first region, the preference function can be defined by an exponential function:

$$
p_{i}^{1}=p_{i 1} \exp \left[\left(\frac{s_{i}^{1}}{p_{i 1}}\right)\left(f_{i}-f_{i 1}\right)\right]
$$

\subsection{Fuzzy preference}

To take into account the decision maker's physical understanding of the desired design outcomes, a fuzzy preference is introduced during the optimization process. In this way, it can enable the decision maker to control the optimization to some extent. Suppose that the preference function of the $i-t h$ objectives belongs to Class- 1 , we define the parameter $f_{i k}$ as a normal fuzzy number $\tilde{f_{i k}}$ and therefore, its membership function follows the form:

$$
\mu_{\tilde{f_{i k}}}\left(f_{i}\right)=\exp ^{-\left[\frac{f_{i}-f_{i k}}{\delta_{i k}}\right]^{2}}, \quad \delta_{i k}>0
$$

where $\delta_{i k}$ is the fuzzy parameter of the $k-t h$ boundary of preference function and it can be defined based on [25, 26. Then the fuzzy preference function can be summarised as follows:

$$
f_{p_{i k}}\left(\tilde{f_{i k}}\right)=\frac{\int_{f_{i}(X)-3 \delta_{i k}}^{f_{i}(X)+3 \delta_{i k}} \bar{f}_{i}\left(f_{i}\right) \mu_{\tilde{f_{i k}}}\left(f_{i}\right) d f_{i}}{\int_{f_{i}(X)-3 \delta_{i k}}^{f_{i}(X)+3 \delta_{i k}} \mu_{\tilde{f_{i k}}}\left(f_{i}\right) d f_{i}}
$$


$\bar{f}_{i}\left(f_{i}\right)$ is the preference function of $i-t h$ objective function without considering fuzzy factor.

\subsection{Fuzzy programming problem}

By defining the fuzzy preference function and range parameters for each objective function, the compromise solution can be achieved by solving the optimization problem as follows:

$$
\begin{cases}\min P=\log _{10}\left\{\frac{1}{n_{s}} \sum_{i=1}^{n_{s}} f_{p_{i k}}\left(\tilde{f_{i k}}\right)\right\}, & \\ f_{i}(X) \leq f_{i 5}, & \text { for class 1-S; } \\ f_{i}(X) \geq f_{i 5}, & \text { for class 2-S; } \\ f_{i 5 L} \leq f_{i}(X) \geq f_{i 5 R}, & \text { for class 3-S; } \\ f_{i 5 L} \leq f_{i}(X) \geq f_{i 5 R}, & \text { for class 4-S. }\end{cases}
$$

where $n_{s}$ is the number of ranges associated with the problem and the range as an example, the fuzzy preference function is shown in Fig.2.

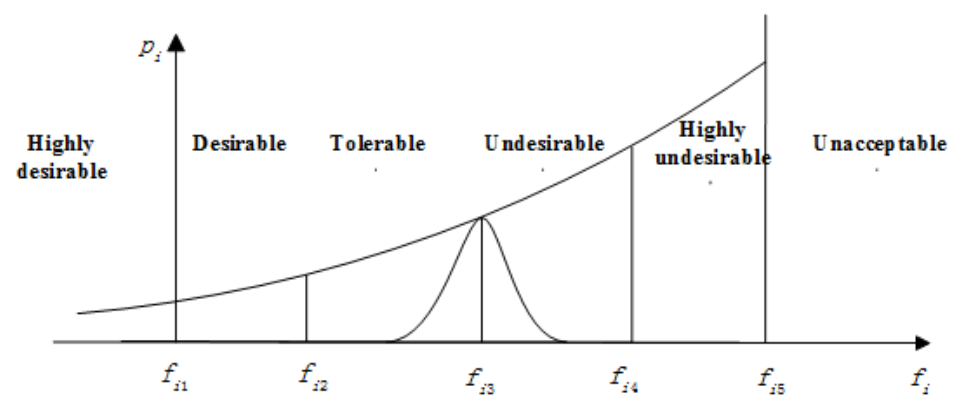

Figure 2: Fuzzy preference function for Class 1-S

\section{Simulation results}

\subsection{Parameters setting}

The maximum values of heat flux, dynamic pressure and load factor are ${ }_{255} Q_{d \max }=200 \mathrm{Btu} / \mathrm{ft} \mathrm{t}^{2} / \mathrm{s} ; P_{d \max }=13406.4583 \mathrm{~Pa} ; n_{\text {Lmax }}=2.5$, respectively. The initial and terminal conditions of the entire process and boundary constraints can be found in Table 2 . 
Table 2: Parameters setting

\begin{tabular}{c|c|c|c|c|c}
\hline \hline & Initial & Bottom & Terminal & Minimum & Maximum \\
\hline $\mathrm{r}(\mathrm{ft})$ & 21162900 & 21066900 & 21162900 & 21066900 & 21162900 \\
$\theta(\mathrm{deg})$ & 0 & free & free & -180 & 180 \\
$\phi(\mathrm{deg})$ & 0 & free & free & -70 & 70 \\
$\mathrm{~V}(\mathrm{ft} / \mathrm{s})$ & 25600 & free & free & 2000 & 45000 \\
$\gamma($ deg $)$ & -1 & free & free & -80 & 80 \\
$\psi($ deg $)$ & 90 & free & free & -180 & 180 \\
$\mathrm{~m}(\mathrm{sl})$ & 6309.4 & free & free & 1370.4 & 6309.4 \\
$\alpha($ deg $)$ & 17.43 & free & free & 0 & 40 \\
$\sigma($ deg $)$ & -75 & free & free & -90 & 1 \\
$T(N)$ & 0 & free & free & 0 & 2000000 \\
\hline
\end{tabular}

The specific boundary conditions are given by the industrial sponsor Lockheed Martin Space Systems Company and only the first hop is taken into account in the paper. The initial altitude is around $80 \mathrm{~km}$ where is the edge of atmosphere.

\subsection{Time history of the state and control}

Firstly, the optimization results for each single objective function according to the dynamic model, objectives and constraints given in Section 2 are generated. The results are shown in Figs.3-7.

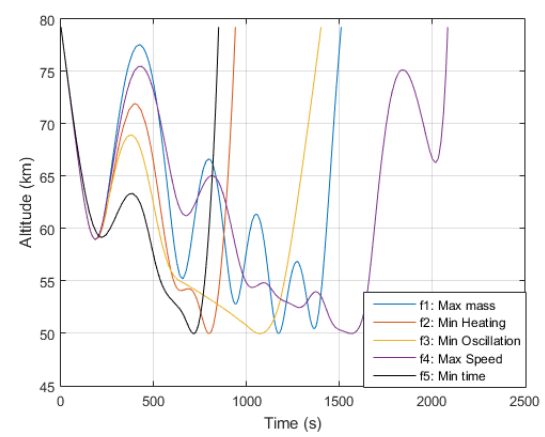

Figure 3: Altitude for different objective function

As can be seen from Fig.3-7, conflicts exist between each objective function, therefore it is impossible to find a solution optimizing each cost function. For 


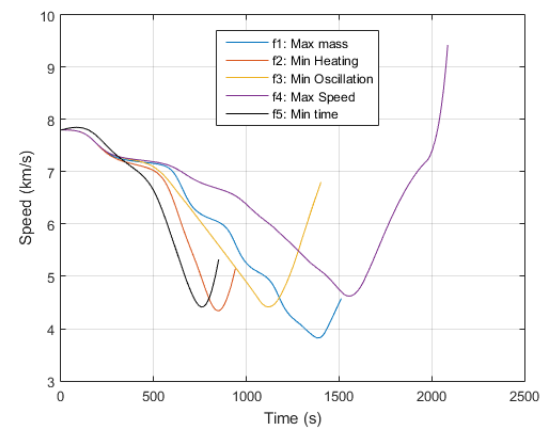

Figure 4: Speed for different objective function

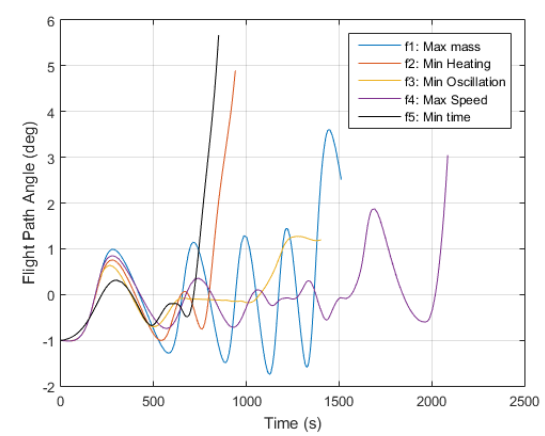

Figure 5: Flight path angle for different objective function

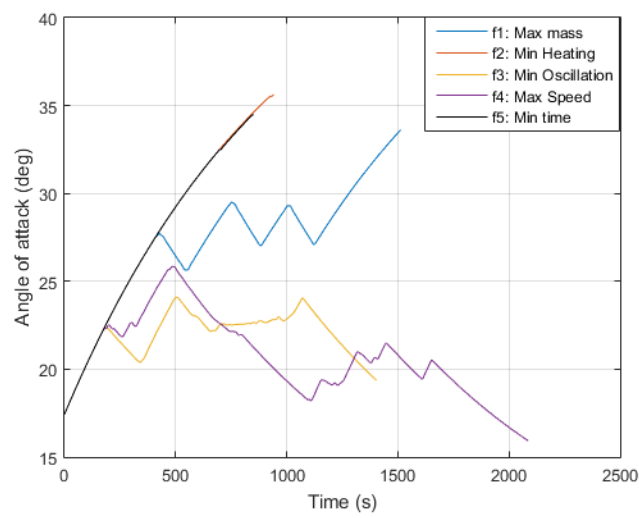

Figure 6: Angle of attack for different objective function 


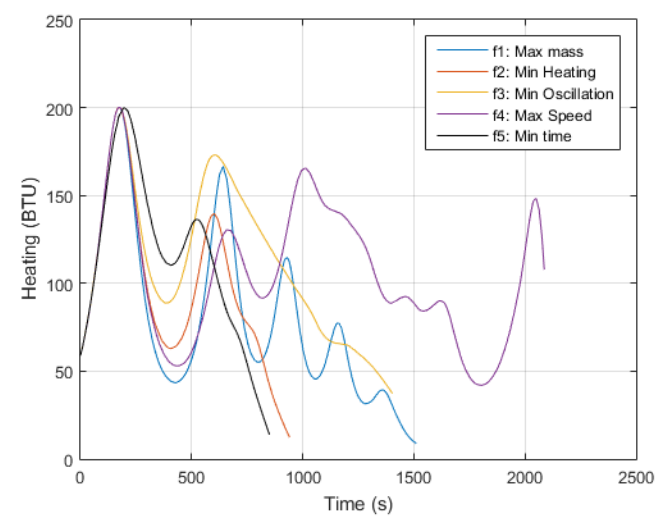

Figure 7: Aerodynamic heating for different objective function

example, in the case of maximizing final velocity, if there is no compromised procedure, then to achieve a higher speeds, the SMV is tending to complete the mission with longer time and accelerating during the whole time period, which means it is trying to consume all the fuel. Hence, after reaching the low earth orbit, the SMV has no fuel left to continue the mission. Similar with [27] presented by Hu and Xin, to see the results more clearly, the payoff table results are tabulated in Table.3.

Table 3: Payoff table

\begin{tabular}{c|c|c|c|c|c}
\hline \hline & $J_{1}$ & $J_{2}$ & $J_{3}$ & $J_{4}$ & $J_{5}$ \\
\hline $\max J_{1}$ & 4437.5 & 121.86 & $8.840 \times 10^{-3}$ & 15013.5 & 1512.04 \\
\hline $\min J_{2}$ & 3971.2 & 75.38 & $2.459 \times 10^{-2}$ & 16887.4 & 940.96 \\
\hline $\min J_{3}$ & 1667.7 & 195.12 & $7.443 \times 10^{-6}$ & 22327.3 & 1403.22 \\
\hline $\max J_{4}$ & 1370.4 & 217.63 & $9.376 \times 10^{-3}$ & 30937.6 & 2086.42 \\
\hline $\min J_{5}$ & 2782.1 & 89.17 & $7.642 \times 10^{-5}$ & 17467.5 & 850.56 \\
\hline
\end{tabular}

From the pay-off table, we can conclude the boundary based on the best solution and worst solution from single objectives results that: $f_{11}=4427.5$, $f_{21}=75.38, f_{31}=7.443 \times 10^{-6}, f_{41}=30937.6, f_{51}=850.56$ and $f_{15}=1370.4$, $f_{25}=217.63, f_{35}=2.459 \times 10^{-2}, f_{45}=15013.5, f_{55}=2086.42$. Then the FFP 
model can be obtained as follows:

$$
\left\{\begin{array}{c}
\min \quad \log _{10}\left\{\frac{1}{5} \sum_{i=1}^{5} f_{p_{i k}}\left(\tilde{f_{i k}}\right)\right\}, \\
f_{1}(X) \geq 1370.4 \\
f_{2}(X) \leq 217.63 \\
f_{3} \leq 2.459 \times 10^{-2} \\
f_{4} \geq 15013.5 \\
f_{5} \leq 2086.42 \\
\dot{Q}_{d}=K_{Q} \rho^{0.5} V^{3.07}\left(c_{0}+c_{1} \alpha+c_{2} \alpha^{2}+c_{3} \alpha^{3}\right)<200 B T U / f t^{2} / s \\
P_{d}=\frac{1}{2} \rho V^{2}<13406.4583 P a \\
n_{L}=\frac{\sqrt{L^{2}+D^{2}}}{m g}<2.5 \\
44,(5),(7) \text { and } 8)
\end{array}\right.
$$

where the first equation of the optimization objective of 29 is to optimize all the objectives by minimizing the preference function values. By solving the model illustrated above, we can achieve the compromised time history of each state and control. The corresponding trajectories generated by using PP and FPP for optimization parameters and constraint can be found in Figs.8-16, respectively.

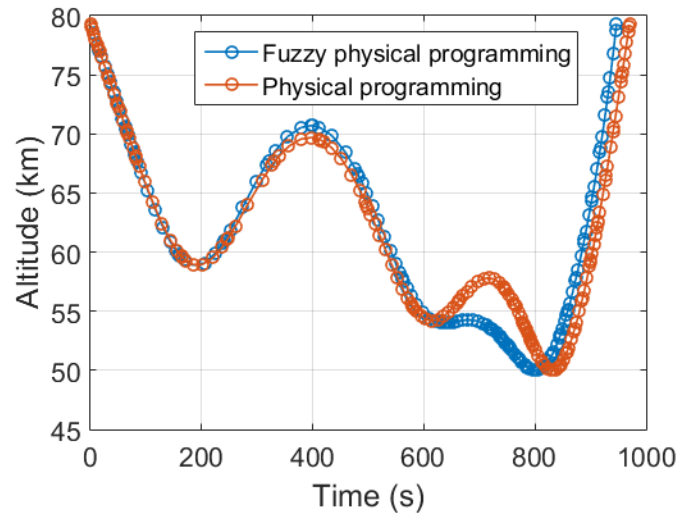

Figure 8: Altitude for FPP and PP

To further compare the results generated from PP and FPP, simulation results were done in order to verify the Pareto optimality of PP and FPP solution by comprising with the Pareto-optimal solutions obtained using NSGA-II and the weighted method, which can be seen in Table 5. NSGA-II shares a simi- 


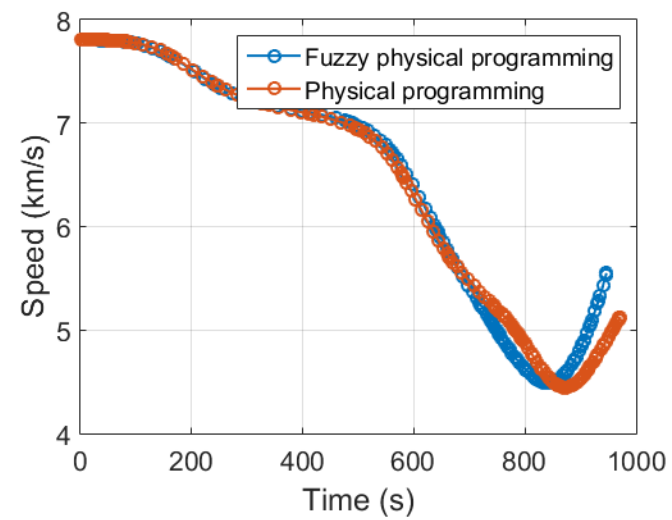

Figure 9: Speed for FPP and PP

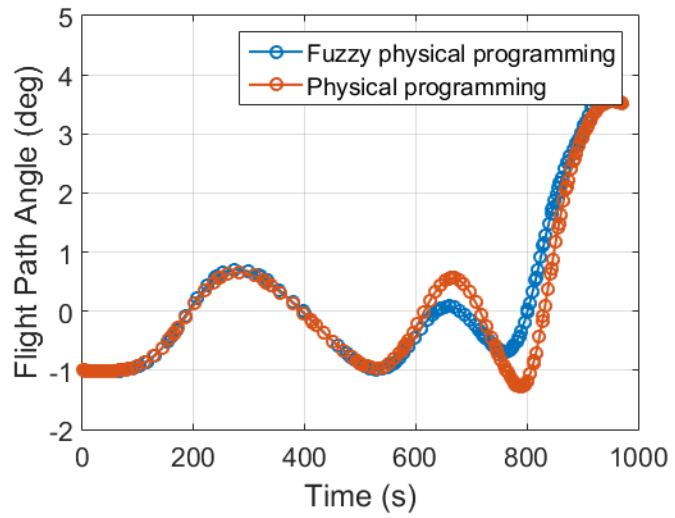

Figure 10: Flight path angle for FPP and PP

290 fitness function and creates a number of fronts. During the optimization procedure, the first front is generated as the set of solutions that has the highest fitness value and is not dominated by any other solutions in the current population. For the NSGA-II simulation, the population size is 200, the maximum number of generations is 500 while the tournament selection scale is 3 . The probability of crossover and mutation are 0.8 and 0.1 , respectively.

The Pareto fronts, generated by NSGA-II, PP, FPP and weighted method(i.e. solutions can be found in Table.4), are projected onto two plane shown in Fig.17 and Fig.18. From the plane of minimizing terminal time versus minimizing to- 


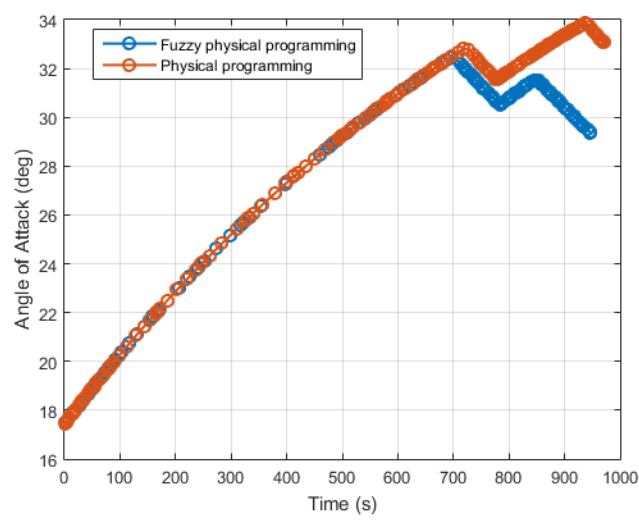

Figure 11: Angle of attack FPP and PP

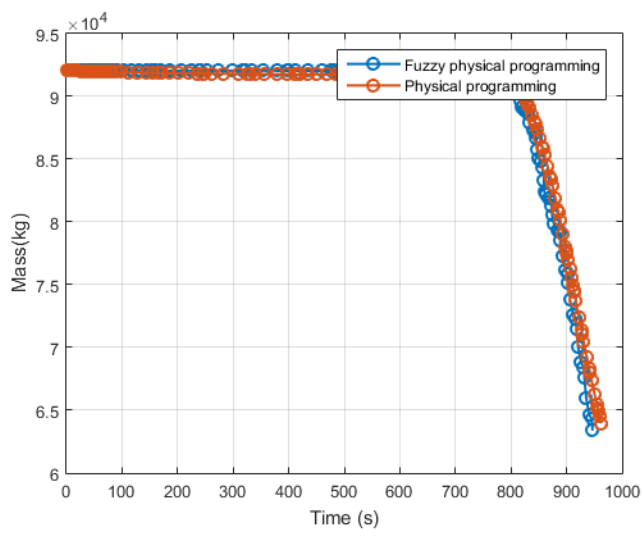

Figure 12: Mass for FPP and PP

Table 4: Optimization results for weighted method

\begin{tabular}{cc}
\hline \hline Weights & Objective values $\left(J_{1}, J_{2}, J_{3}, J_{4}, J_{5}\right)$ \\
\hline$(0.30,0.10,0.10,0.30,0.20)$ & $\left(4330.2,97.57,7.93 \times 10^{-4}, 16770.7,963.9\right)$ \\
$(0.35,0.15,0.15,0.20,0.15)$ & $\left(4342.3,99.48,7.63 \times 10^{-4}, 16502.2,975.5\right)$ \\
$(0.40,0.20,0.20,0.10,0.10)$ & $\left(4378.7,105.42,6.75 \times 10^{-4}, 16492.2,988.1\right)$ \\
\hline
\end{tabular}

tal heating and maximizing final velocity versus maximizing final mass, it is clear that both PP and FPP can get the solutions around the Pareto frontier generated by NSGA-II while it is hard for weighted method to generate a Pareto-optimal solution. Specifically, the compromised solution obtained by 


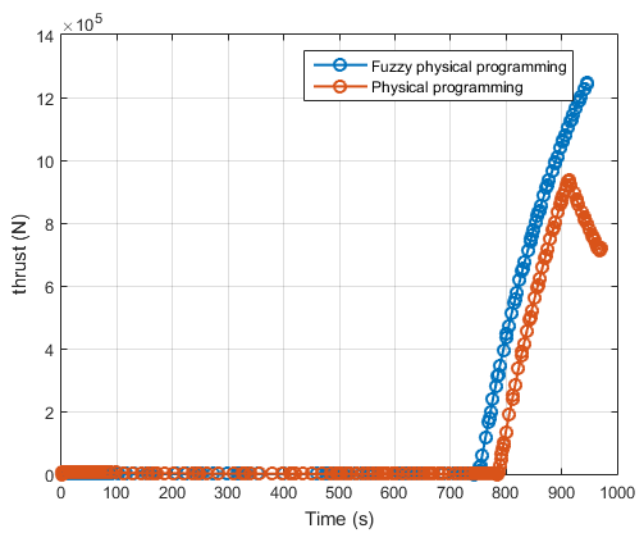

Figure 13: Thrust for FPP and PP

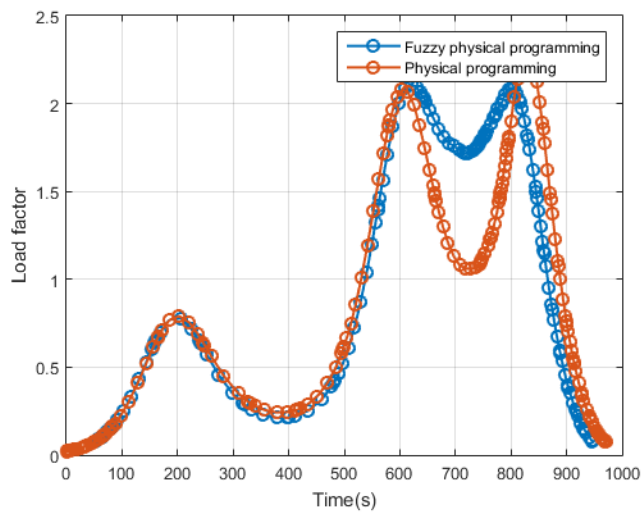

Figure 14: Load factor for different objective function

FPP can have a better preference than its counterpart PP, hence it has smaller variance with the Pareto frontier. As FPP approach is designed to generate a single Pareto-optimal solution in one run whereas NSGA-II approach is designed to obtain hundreds of solutions in one run, therefore, FPP is definitely competitive in computational effort and is more convenient in the numerical experiments and practical use.

Table. 5 contains the compromised solution for each objective function and the variance proportion. As can be seen from Table.5, the maximum variance percentage can reach around $8.4 \%$, that means if we do not consider the fuzzy factor, there will be some differences between the calculated solution with com- 


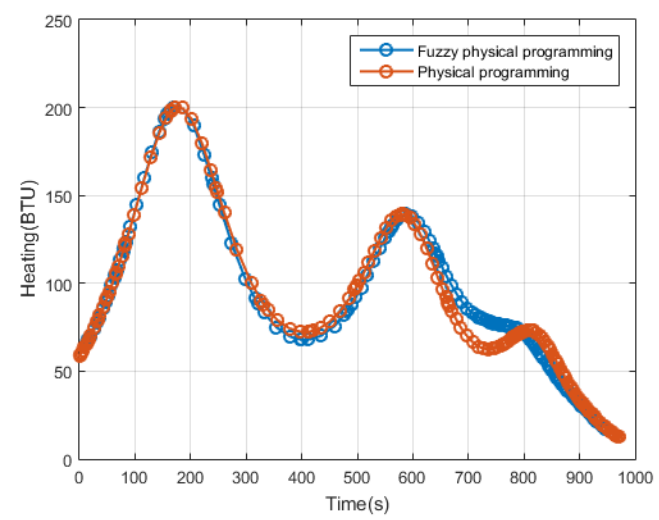

Figure 15: Aerodynamic heating for different objective function

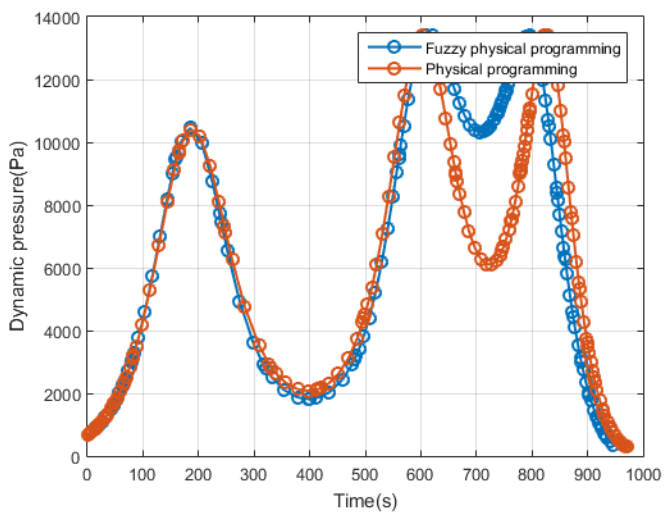

Figure 16: Dynamic pressure for different objective function

pared to the practical solution. Both solutions calculated from PP and FPP satisfy the path constraints and for the solution of FPP, it is found that all of the compromised objective values can be successfully obtained. Specifically, the value of $J_{1}$ is in the tolerable region whereas the value of $J_{2}$ is in the desirable region. $J_{3}$ and $J_{5}$ are in the tolerable region while the value of $J_{4}$ can reach desirable region. This further proves that the combination of hp-adaptive discrete method with FPP can be applied to multi-objective optimization for trajectory design problems.

Using the technique of hp-adaptive pseudospectral method, the time history for the states and controls can be much smoother. This can be seen from 


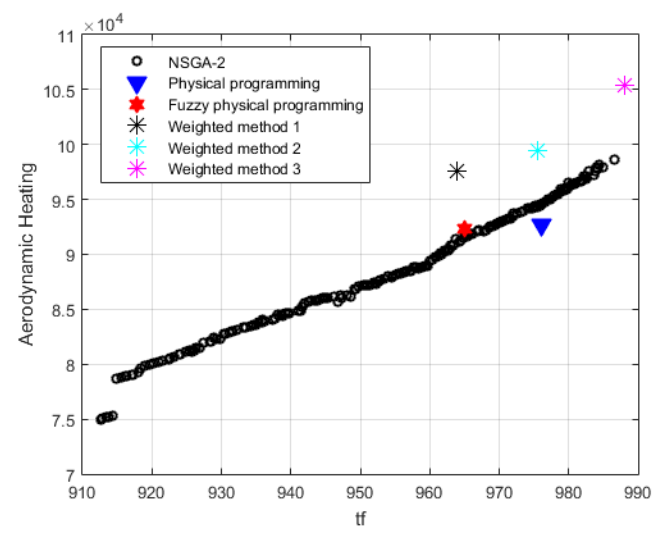

Figure 17: Total heating versus terminal time

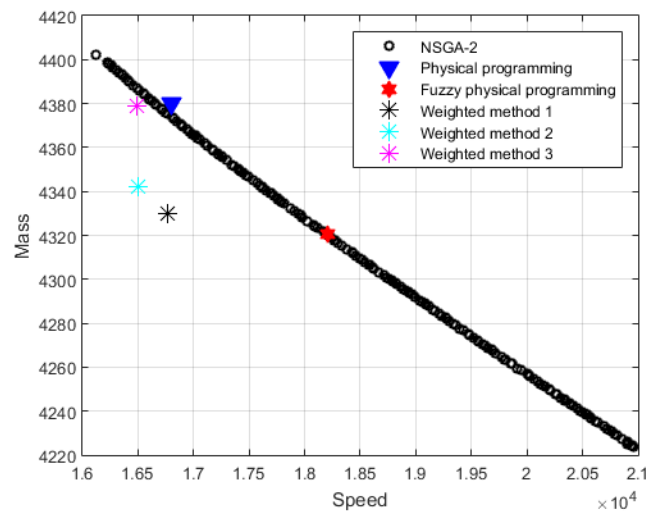

Figure 18: Final mass versus final speed

Table 5: Objective values

\begin{tabular}{c|c|c|c|c|c}
\hline \hline & $J_{1}$ & $J_{2}$ & $J_{3}$ & $J_{4}$ & $J_{5}$ \\
\hline $\mathrm{PP}$ & 4380.3 & 92.63 & $5.43 \times 10^{-4}$ & 16798.5 & 976.06 \\
\hline $\mathrm{FPP}$ & 4330.1 & 92.35 & $5.01 \times 10^{-4}$ & 18208.1 & 965.62 \\
\hline Variance(\%) & 1.6 & 0.3 & 5.9 & 8.4 & 1.1 \\
\hline
\end{tabular}

Figs.8-16, where the distribution of collocation points tend to be denser at those 325 areas having a high value of curvature, on the other hand, the distribution of collocation points tend to be sparser at those flat areas. That way can control the number of optimization parameters and control the size of NLP problem 
effectively. Also, since the trajectory becomes smoother, it is more possible and reasonable for practical use.

The accumulation of errors generated from numerical simulation model should be evaluated depending on technique used. The parameters used in model have errors and are modeled in the simulation as a Gaussian white noises. Comparative simulation results are shown in Fig.19-20. It is shown that computational errors accumulation affects the shape of trajectory slightly. The reliability of results can be guaranteed since the hp-strategy used for mesh refinement can improve the accuracy of current mesh grids.

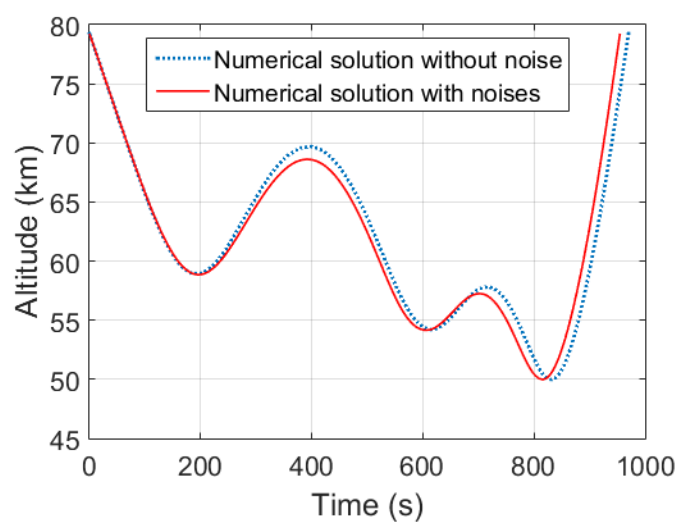

Figure 19: Altitude vs time

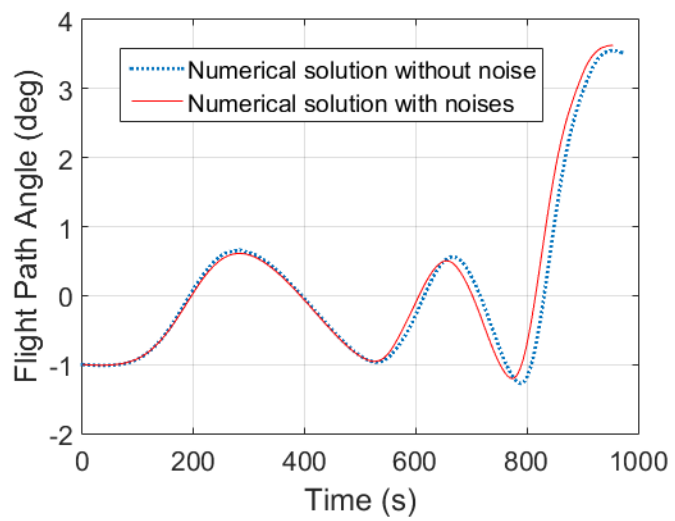

Figure 20: Flight path angle vs time

In summary, all the figures and data provided earlier confirm the feasibility 
of the proposed combination method in terms of SMV trajectory design problem.

\section{Conclusions}

[3] A. Rajesh, Reentry Trajectory Optimization: Evolutionary Approach,
Multidisciplinary Analysis Optimization Conferences, American Institute
of Aeronautics and Astronautics, doi:10.2514/6.2002-5466. doi:doi:10.

[3] A. Rajesh, Reentry Trajectory Optimization: Evolutionary Approach,
Multidisciplinary Analysis Optimization Conferences, American Institute
of Aeronautics and Astronautics, doi:10.2514/6.2002-5466. doi:doi:10.

In order to design a proper trajectory for the SMV, the previously proposed single-objective optimization formulation were extended to multi-objective optimization, including maximum final mass, minimum total aerodynamic heating, minimum oscillation, maximum final velocity and minimum final time. A multi-objective optimization method FPP based on hp-adaptive pseudospectral method was proposed. When considering the fuzzy factor for the system, in FPP, fuzzy preference function is introduced to adjust the boundary defined by the decision maker. The simulation results show that there are significant differences between each single-objective solution and by using the FFP method, a compromised solution can be obtained. A comparison was made between the sesults obtained by using FPP and NSGA-II, which showed that the method proposed in this paper can have a better preference in terms of generating Pareto optimal solution than other methods and therefore, it tends to be feasible for the SMV trajectory design problem and the definition of the fuzzy function is important.

\section{References}

[1] C. Gan, W.Zi-ming, X. Min, C. Si-lu, Genetic Algorithm Optimization of RLV Reentry Trajectory, International Space Planes and Hypersonic Systems and Technologies Conferences, American Institute of Aeronautics and As-

tronautics, doi:10.2514/6.2005-3269. doi:doi:10.2514/6.2005-326910. 2514/6.2005-3269.

[2] Z. Kenan, C. Wanchun, Reentry Vehicle Constrained Trajectory Optimization, International Space Planes and Hypersonic Systems and Technologies Conferences, American Institute of Aeronautics and Astronautics, doi:10.2514/6.2011-2231. doi:doi:10.2514/6.2011-223110.2514/ 6.2011-2231. 2514/6.2002-546610.2514/6.2002-5466. 

mum heating reentry trajectories for advanced hypersonic launch vehicles, Guidance, Navigation, and Control and Co-located Conferences, American Institute of Aeronautics and Astronautics, doi:10.2514/6.1997-3535. doi:doi:10.2514/6.1997-353510.2514/6.1997-3535.

375 [5] T. Lips, B. Fritsche, A comparison of commonly used re-entry analysis tools, Acta Astronautica 57 (2) (2005) 312-323.

[6] M. Reyhanoglu, J. Alvarado, Estimation of debris dispersion due to a space vehicle breakup during reentry, Acta Astronautica 86 (3) (2013) 211-218.

[7] J. T. Betts, Survey of numerical methods for trajectory optimization, Journal of Guidance, Control, and Dynamics 21 (2) 193-207. doi:10.2514/2. 4231.

[8] I. Mikhail, V. Pavel, K. Alexandr, Numerical Investigation of the EXPERT Reentry Vehicle Aerothermodynamics Along the Descent Trajectory, Fluid Dynamics and Co-located Conferences, American Institute of Aeronautics and Astronautics, doi:10.2514/6.2007-4145. doi:doi:10.2514/6. 2007-414510.2514/6.2007-4145.

[9] G. Peter, W. Klaus, Trajectory optimization using a combination of direct multiple shooting and collocation, Guidance, Navigation, and Control and Co-located Conferences, American Institute of Aeronautics and Astronautics, doi:10.2514/6.2001-4047. doi:doi:10.2514/6.2001-404710. 2514/6.2001-4047.

[10] G. W. Reddien, Collocation at gauss points as a discretization in optimal control, SIAM Journal on Control and Optimization 17 (2) 298-306. doi: doi:10.1137/0317023

[11] F. Fariba, I. Ross, Costate estimation by a Legendre pseudospectral method, Guidance, Navigation, and Control and Co-located Conferences, American Institute of Aeronautics and Astronautics, doi:10.2514/6.19984222. doi:doi:10.2514/6.1998-422210.2514/6.1998-4222

[12] S. Jain, P. Tsiotras, Trajectory optimization using multiresolution techniques, Journal of Guidance, Control, and Dynamics 31 (5) 1424-1436. doi:10.2514/1.32220 
[13] R. Jeremy, Launch Vehicle Trajectory Optimization Using a Legendre Pseudospectral Method, Guidance, Navigation, and Control and Colocated Conferences, American Institute of Aeronautics and Astronautics, doi:10.2514/6.2003-5640. doi:doi:10.2514/6.2003-564010.2514/6. 2003-5640.

[14] D. A. Benson, G. T. Huntington, T. P. Thorvaldsen, A. V. Rao, Direct trajectory optimization and costate estimation via an orthogonal collocation method, Journal of Guidance, Control, and Dynamics 29 (6) 1435-1440. doi:10.2514/1.20478.

[15] D. W. Bruno, Generation of pseudospectral differentiation matrices i, SIAM घ Journal on Numerical Analysis 34 (4) 1640-1657. doi:doi:10.1137/ S0036142993295545

[16] J. Timothy, S. Christopher, F. Franklin, R. Anil, Constrained Trajectory Optimization Using Pseudospectral Methods, Guidance, Navigation, and Control and Co-located Conferences, American Institute of Aeronautics and Astronautics, doi:10.2514/6.2008-6218. doi:doi:10.2514/6. 2008-621810.2514/6.2008-6218.

[17] D. Christopher, H. William, R. Anil, An Improved Adaptive hp Algorithm using Pseudospectral Methods for Optimal Control, Guidance, Navigation, and Control and Co-located Conferences, American Institute of Aeronautics and Astronautics, doi:10.2514/6.2010-8272. doi:doi:10.2514/6. 2010-827210.2514/6.2010-8272.

[18] C. L. Darby, W. W. Hager, A. V. Rao, Direct trajectory optimization using a variable low-order adaptive pseudospectral method, Journal of Spacecraft and Rockets 48 (3) 433-445. doi:10.2514/1.52136.

[19] G. Divya, H. William, R. Anil, Gauss Pseudospectral Method for Solving Infinite-Horizon Optimal Control Problems, Guidance, Navigation, and Control and Co-located Conferences, American Institute of Aero430 ㅁ nautics and Astronautics, doi:10.2514/6.2010-7890. doi:doi:10.2514/6. 2010-789010.2514/6.2010-7890.

[20] C.-H. Huang, J. Galuski, C. L. Bloebaum, Multi-objective pareto concur- 
rent subspace optimization for multidisciplinary design, AIAA Journal 45 (8) 1894-1906. doi:10.2514/1.19972.

435

440

口

[24] N. N. Smirnov, V. B. Betelin, V. F. Nikitin, Y. G. Phylippov, J. Koo, Deto-

[21] K.-P. Lin, Y.-Z. Luo, G.-J. Tang, Multi-objective optimization of space station logistics strategies using physical programming, Engineering Optimization 47 (8) 1140-1155. doi:10.1080/0305215X.2014.954568

[22] A. Messac, Physical programming - effective optimization for computational design, AIAA Journal 34 (1) 149-158. doi:10.2514/3.13035.

[23] N. N. Smirnov, V. B. Betelin, V. F. Nikitin, L. I. Stamov, D. I. Altoukhov, Accumulation of errors in numerical simulations of chemically reacting gas dynamics, Acta Astronautica 117 338-355. doi:http://dx.doi.org/10. 1016/j.actaastro.2015.08.013. nation engine fed by acetylene-oxygen mixture, Acta Astronautica 104 (1) 134-146. doi:http://dx.doi.org/10.1016/j.actaastro.2014.07.019.

[25] R. V. Tappeta, J. E. Renaud, A. Messac, G. J. Sundararaj, Interactive physical programming: Tradeoff analysis and decision making in multicriteria optimization, AIAA Journal 38 (5) 917-926. doi:10.2514/2.1048.

[26] X. Zhang, H.-Z. Huang, L. Yu, Fuzzy preference based interactive fuzzy physical programming and its application in multi-objective optimization, Journal of mechanical science and technology 20 (6) (2006) 731-737.

[27] C.-F. Hu, Y. Xin, Reentry trajectory optimization for hypersonic vehicles using fuzzy satisfactory goal programming method, International Journal of Automation and Computing 12 (2) (2015) 171-181. 


\title{
Fuzzy physical programming for Space
} Manoeuvre Vehicles trajectory optimization based on hp-adaptive pseudospectral method

\author{
Chai, Runqi
}

Elsevier

Runqi Chai, Al Savvaris and Antonios Tsourdos, Fuzzy physical programming for Space Manoeuvre Vehicles trajectory optimization based on hp-adaptive pseudospectral method, Acta pÿAstronautica, Volume 123, June July 2016, pp62-70

http://dx.doi.org/10.1016/j.actaastro.2016.02.020

Downloaded from Cranfield Library Services E-Repository 